\title{
Cross-modal enhancement of the MMN to speech- sounds indicates early and automatic integration of letters and speech-sounds
}

Citation for published version (APA):

Froyen, D. J. W., van Atteveldt, N. M., Bonte, M. L., \& Blomert, L. P. M. (2008). Cross-modal enhancement of the MMN to speech-sounds indicates early and automatic integration of letters and speech-sounds. Neuroscience Letters, 430(1), 23-28. https://doi.org/10.1016/j.neulet.2007.10.014

Document status and date:

Published: 01/01/2008

DOI:

10.1016/j.neulet.2007.10.014

Document Version:

Publisher's PDF, also known as Version of record

Document license:

Taverne

Please check the document version of this publication:

- A submitted manuscript is the version of the article upon submission and before peer-review. There can be important differences between the submitted version and the official published version of record.

People interested in the research are advised to contact the author for the final version of the publication, or visit the DOI to the publisher's website.

- The final author version and the galley proof are versions of the publication after peer review.

- The final published version features the final layout of the paper including the volume, issue and page numbers.

Link to publication

\footnotetext{
General rights rights.

- You may freely distribute the URL identifying the publication in the public portal. please follow below link for the End User Agreement:

www.umlib.nl/taverne-license

Take down policy

If you believe that this document breaches copyright please contact us at:

repository@maastrichtuniversity.nl

providing details and we will investigate your claim.
}

Copyright and moral rights for the publications made accessible in the public portal are retained by the authors and/or other copyright owners and it is a condition of accessing publications that users recognise and abide by the legal requirements associated with these

- Users may download and print one copy of any publication from the public portal for the purpose of private study or research.

- You may not further distribute the material or use it for any profit-making activity or commercial gain

If the publication is distributed under the terms of Article $25 \mathrm{fa}$ of the Dutch Copyright Act, indicated by the "Taverne" license above, 


\title{
Cross-modal enhancement of the MMN to speech-sounds indicates early and automatic integration of letters and speech-sounds
}

\author{
Dries Froyen*, Nienke Van Atteveldt, Milene Bonte, Leo Blomert \\ Department of Cognitive Neuroscience, Faculty of Psychology, University of Maastricht, \\ Maastricht Brain Imaging Center (M-BIC), Maastricht, The Netherlands
}

Received 22 December 2006; received in revised form 10 July 2007; accepted 15 October 2007

\begin{abstract}
Recently brain imaging evidence indicated that letter/speech-sound integration, necessary for establishing fluent reading, takes place in auditory association areas and that the integration is influenced by stimulus onset asynchrony (SOA) between the letter and the speech-sound. In the present study, we used a specific ERP measure known for its automatic character, the mismatch negativity (MMN), to investigate the time course and automaticity of letter/speech-sound integration. We studied the effect of visual letters and SOA on the MMN elicited by a deviant speech-sound. We found a clear enhancement of the MMN by simultaneously presenting a letter, but without changing the auditory stimulation. This enhancement diminishes linearly with increasing SOA. These results suggest that letters and speech-sounds are processed as compound stimuli early and automatically in the auditory association cortex of fluent readers and that this processing is strongly dependent on timing.
\end{abstract}

(c) 2007 Elsevier Ireland Ltd. All rights reserved.

Keywords: Letter/speech-sound integration; Mismatch negativity; Time-window for integration

Learning to read is a multistage process that presupposes the establishment of associations between letters and speechsounds. Most models describing the process of learning to read emphasize the generation of letter to speech-sound connections as a crucial first step in the learning process [5]. At least $90 \%$ of school children learn the relation between letter/speech-sound pairs within a few months without exceptional effort [2]. At the same time, we also know that failure of this step in reading acquisition is considered a main cause for reading problems like in developmental dyslexia [18]. Snowling [15] showed that dyslexic readers revealed a weaker developmental performance increase on a letter/speech-sound matching task in comparison with their normally reading peers, indicating that fluent reading probably requires full automatization of letter/speech-sound associations.

Despite the importance for acquiring fluent reading skills, the behavioral and neural characteristics of letter/speech-sound associations have hardly been investigated. Dijkstra et al. tested the influence of congruent and incongruent letter primes on the

\footnotetext{
* Corresponding author at: Department of Cognitive Neuroscience, Faculty of Psychology, University of Maastricht, P.O. Box 616, 6200 MD Maastricht, The Netherlands. Tel.: +31 4338 82205; fax: +31 433884125 .

E-mail address: d.froyen@ psychology.unimaas.nl (D. Froyen).
}

performance in a speech-sound monitoring task. The results revealed faster identification of congruent in comparison with incongruent letter primes, thus indicating automatic influences of letters on speech-sound processing [4]. Raij et al. [12] conducted a magneto-encephalography (MEG) study to investigate the neural time course of letter/speech-sound integration. Simultaneously presented congruent letters and speech-sounds suppressed brain activations in the superior temporal sulcus (STS) around $345 \mathrm{~ms}$ after stimulus onset in comparison with the sum of brain activations in response to the unimodal stimuli, indicating letter/speech-sound integration in this area. Recently, letter/speech-sound processing was investigated with fMRI to obtain a more precise localization of the neural mechanisms involved in their integration $[16,17]$. The results of a first fMRI study showed that not only STS and superior temporal gyrus (STG) responding to heteromodal stimuli, but also auditory association cortex (planum temporale, PT) responding only to auditory stimuli, were crucially involved in the neural binding of letters and speech-sounds [17]. Besides enhanced activation in response to congruent and incongruent presentations in STS and STG, enhancement for congruent and suppression for incongruent presentations were found in PT. This result was replicated in a subsequent study in which the stimulus onset asynchrony (SOA) between letters and speech-sounds 
was varied [16]. The results revealed significant interactions between SOA and congruency in the PT, indicating temporal synchrony to be critical for the integration of letters and speech-sounds in this auditory area. The modulation of auditory processing by letters in the PT occurred within a very narrow time-window with already declining influence at $150 \mathrm{~ms}$ SOA.

In sum, letter/speech-sound integration seems to be performed by a network of the PT and the STS/STG, and seems dependent on a narrow time-window of integration. Given the passive nature of the tasks used in the fMRI studies (passive viewing/listening), letter/speech-sound integration seems to occur automatically.

In the present study, we further investigate the automaticity and SOA-dependency of letter/speech-sound integration. We used the mismatch negativity (MMN), because it is evoked early and automatically [9]. This allows investigating the automatic character of letter/speech-sound associations with high temporal accuracy. If in a sequence of auditory stimuli a rarely presented sound (the deviant) deviates in one or more aspects from the sound that is frequently presented (the standard), an MMN is evoked between 100 and $200 \mathrm{~ms}$ after stimulus onset. The MMN is considered to reflect the neurophysiological correlate of a comparison process between an incoming auditory stimulus and the memory trace formed by the repetitive aspect of the standard stimulus [14]. It is generally assumed that a major MMN source is located in the primary auditory cortex, which might be related to the sensory memory mechanism, but additional frontal generators have been described [1,7]. The MMN has repeatedly been shown to be sensitive to phonemic processing [10].

In the present report, we presented an oddball paradigm with speech-sounds in two different contexts. We compared the MMN evoked by deviant speech-sounds (auditory experiment) with the MMN evoked by the same deviant speech-sounds combined with visually presented letters (audiovisual experiment). We conducted four different experiments, one auditory and three audiovisual experiments. In all four experiments, subjects passively listened to the speech-sounds /a/(standard, 90\%) and $/ \mathrm{o} /($ deviant, $10 \%)$. In the auditory experiment, subjects listened to these speech-sounds while watching a silent movie. In the three audiovisual experiments, subjects listened to the same speech-sounds, while watching single letters. The three audiovisual experiments differ only with regard to the stimulus onset asynchrony between the presentation of the letter and speech-sound. The letter always appeared first and the speechsound respectively, 0,100 or $200 \mathrm{~ms}$ later. We will from now on refer to the three audiovisual experiments as AV_0, AV_100 and AV_200.

We first hypothesized that the deviant speech-sound would elicit a typical MMN (auditory experiment). Second, we expected that the letter/speech-sound pair is automatically processed as a compound stimulus in the auditory cortex. As a consequence, we hypothesized that the presence of a visual letter may influence the MMN response to the deviant speech-sound (audiovisual experiment). Third, if there is an influence, we predicted that it may be sensitive to a temporal asynchrony between the letter and speech-sound as indicated by the strong SOA effect found in Van Atteveldt et al. [16]. We chose 100 and $200 \mathrm{~ms}$
SOA since these SOAs include the significantly effective SOA of $150 \mathrm{~ms}$ used in Van Atteveldt et al. [16]. The fact that the major MMN source is located in the early auditory cortex [1,7], including the planum temporale where the reported effect of SOA was most pronounced [16], underpinned our prediction.

Fourteen subjects participated in the auditory experiment (11 female, range 18-33, mean age 21.92 years). Twelve subjects participated in AV_0 (nine females, range 20-33, mean age 24.69 years). Thirteen subjects participated in AV_100 (nine females, range 19-29, mean age 22.38 years). Seventeen subjects participated in AV_200 (13 females, range 18-33, mean age 23.05 years). Three subjects participated in all four experiments. Another 11 subjects participated both in the auditory experiment and in AV_200. Subjects gave informed written consent and were paid for their participation. All subjects were undergraduate university students without any history of hearing or reading problems and with normal or corrected to normal vision. One subject was left handed and participated in the auditory experiment and in AV_200.

Stimuli were natural speech-sounds, /a/ (384 ms) and /o/ (348 ms), and the visually presented single letter "a". Speechsounds were digitally recorded (sampling rate $44.1 \mathrm{kHz}, 16$ bit quantization) from a female speaker. Recordings were bandpass filtered (180-10.000 Hz) and resample at $22.05 \mathrm{kHz}$ and matched for loudness with Praat software [3]. The sounds were presented binaurally through loudspeakers at about $65 \mathrm{~dB}$ SPL. The letter was presented in white on a black background in the centre of a computer screen for $500 \mathrm{~ms}$, printed in lower case font "Arial" at letter size 40. Between the presentations of the stimuli, a white fixation cross was presented in the centre of the screen. To ensure that subjects were at all times focusing the screen, we interspersed the experimental trials with a non-related color picture. Subjects had to press a button when they saw the picture. Since the focusing task was very easy and not related to the stimuli of interest we suggest that this did not influence the MMN, a very basic deviance detection mechanism of the brain [14], problematically. Each experiment consisted of four experimental blocks with 534 trials. Trial length was always $1250 \mathrm{~ms}$. When subjects heard the speech-sound deviant /o/, we always presented the standard letter "a". Although we also manipulated the visual stimuli in some trials, these conditions are not relevant for the present study and are reported elsewhere [6].

EEG data were recorded with NeuroScan 4.2 from 0.01 to $50 \mathrm{~Hz}$ with a sampling rate of $250 \mathrm{~Hz}$ in a sound-attenuating and electrically shielded room from 30 electrode positions (Extended International 10-20 system) relative to a nose reference. Eye-movements and blinks were measured with bipolar VEOG/HEOG channels. All electrode impedance levels (EEG and EOG) were kept below $5 \mathrm{k} \Omega$. EEG data were epoched from -50 to $850 \mathrm{~ms}$ relative to stimulus onset, baseline corrected $(50 \mathrm{~ms}$ pre-stimulus interval), and $1-30 \mathrm{~Hz}$ band pass filtered with NeuroScan 4.2. Epochs containing data exceeding a maximum voltage criterion of $75 \mu \mathrm{V}$ were rejected. Standards immediately following deviants were not included in the analyses, as were the trials with target pictures. The raw EEG data were corrected for vertical eye-movements (i.e. blink artifacts). In the auditory experiment, we included 146 (73\%) of 
the standard trials and $151(75 \%)$ of the deviant trials. In AV_0, we retained respectively, $184(92 \%)$ and $185(93 \%)$ of the trials, in AV_100 $182(91 \%)$ and $186(93 \%)$ and in AV_200 180 (90\%) and $182(91 \%)$. Epochs were averaged separately for each condition and each participant from the onset of the visual stimulus.

Difference waves were calculated by subtracting the ERPs to the standard condition from ERPs to the deviant condition. In the audiovisual experiments, the visual stimulus was always the same in the standard and the deviant conditions (the letter "a"). Therefore, the same visual brain wave will be evoked in both conditions. Since the auditory stimulus was always presented simultaneously with or later than the letter, the visual evoked potentials elicited by the letters can be subtracted out by calculating deviant minus standard difference waves.

To investigate the MMN, statistical analyses were performed on nine electrodes covering frontal, central, centroparietal and temporal areas $(\mathrm{Fz}, \mathrm{Cz}, \mathrm{FC} 3, \mathrm{FC} 4, \mathrm{~T} 3, \mathrm{~T} 4, \mathrm{CP} 3, \mathrm{CP} 4$ and $\mathrm{Pz}$ ), followed by a detailed analysis on the frontocentral electrodes $(\mathrm{Fz}, \mathrm{Cz}, \mathrm{FC} 3, \mathrm{FC} 4)$, where the MMN effect was most prominent. Timing of ERP responses in the MMN window was measured by determining individual peak latencies between 50 and $250 \mathrm{~ms}$ after stimulus onset. This time-window was set after visual inspection of the data and includes the timewindow (100-200 ms) typically reported in MMN studies [14]. Amplitude measures included both individual peak amplitude and mean amplitude taken from the $50 \mathrm{~ms}$ window around the individual peak latency (separately for each subject, condition and electrode). When performing further analysis of the difference waves on the frontocentral electrodes we excluded two subjects with $Z$-scores on all four electrodes higher than 1.96 or lower than -1.96 . We only report results reaching significance at $p<0.05$ after Bonferroni correction.

We first analyzed the auditory experiment to probe a typical MMN evoked by a deviant speech-sound in a standard speechsound context. The left part of Fig. 1 shows the grand average ERPs (A) and difference waveforms (deviant-standard trials) (B) on Fz for the speech-sound deviant in the auditory experi- ment. The speech-sound deviant elicited an MMN with average peak latency of $168 \mathrm{~ms}$ (averaged over the four frontocentral electrodes, range: 94-238 ms) after the onset of the auditory stimulus deviation (Fig. 2), with a topographical distribution that is typically reported (left part Fig. 1C). A polarity reversal of the MMN at electrode sites below the sylvian fissure (left part Fig. 1C) suggests main neural generators in the auditory cortex [14].

Amplitude characteristics of ERP activity in the MMN time-window were first analyzed using a 2 (stimulus type; standard versus deviant) by 9 (electrode sites) repeated-measures ANOVA. We found an interaction between stimulus type and electrode site for mean, $F(8,104)=3.0, p=0.004$, and peak values, $F(8,104)=2.0, p=0.05$. Further analysis was performed on four frontocentral electrodes using a 2 (stimulus type; standard versus deviant) by 4 (electrode sites) repeated-measures ANOVA. There was no interaction with electrode site for mean, $F(3,39)=0.6, p=0.587$, and peak values, $F(3,39)=.6, p=0.58$. We found a marginal effect of stimulus type for mean values, $F(1$, $13)=4.4, p=0.055$ and an effect of stimulus type for peak values, $F(1,13)=5.4, p=0.037$. The deviant grand average waves are systematically more negative in comparison with standard grand average waves. Further analyses of the difference wave were performed with one-sample $t$-tests on each of the frontocentral electrodes, after exclusion of one subject ( $Z$-scores of 2.20 for Fz, 2.15 for $\mathrm{Cz}, 2.0$ for FC3 and 2.1 for FC4). Mean and peak amplitude values were significantly different from zero; for mean amplitudes $\mathrm{Fz}, t(12)=-11.7 ; p<.001, \mathrm{Cz}, t(12)=-10.5$; $p<.001, \mathrm{FC} 3, t(12)=-8.6 ; p<.001$ and FC4, $t(12)=-12.3$; $p<.001$ and for peak amplitudes Fz, $t(12)=-11.7 ; p<.001$, $\mathrm{Cz}, t(12)=-10.5 ; p<.001, \mathrm{FC} 3, t(12)=-8.6 ; p<.001$ and $\mathrm{FC} 4$, $t(12)=-12.3 ; p<.001$.

In all four experiments (one auditory and three audiovisual experiments with different SOAs) the deviant speech-sound stimulus elicited a large negativity around $180 \mathrm{~ms}$ after auditory stimulus onset (rectangle in Fig. 1B), with a typical topographical distribution of the MMN (Fig. 1C). Average peak latencies (averaged over the four frontocentral electrodes)
Auditory experiment

(A)

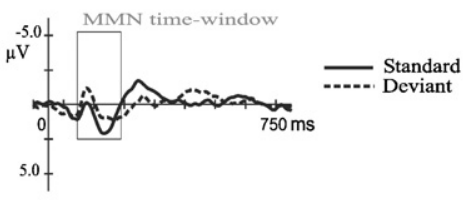

(B)

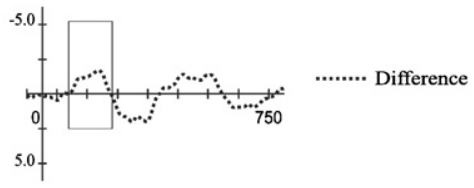

(C)

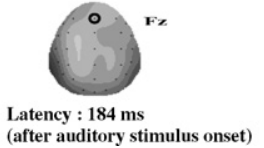

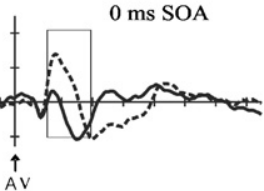
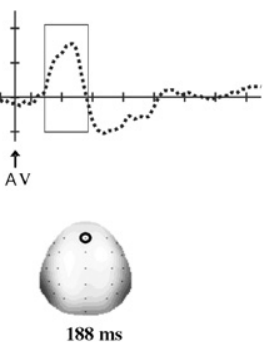

Audiovisual experiments
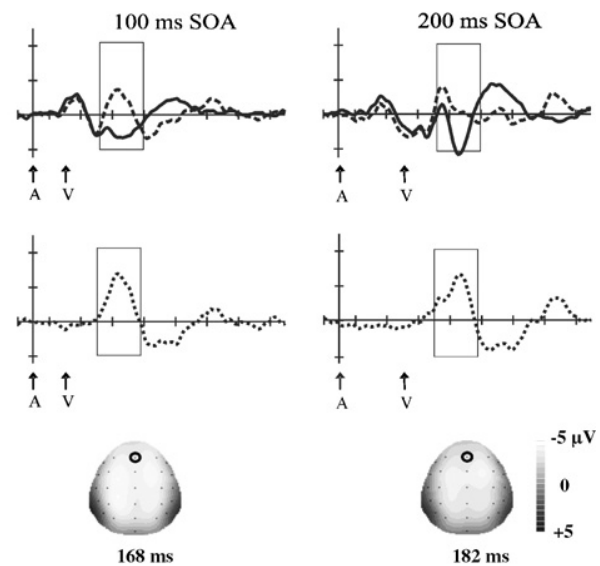

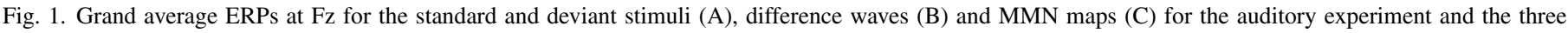
audiovisual experiments. Arrows indicate the onset of visual (V) and auditory (A) stimuli and the rectangle indicates the MMN time-window. 


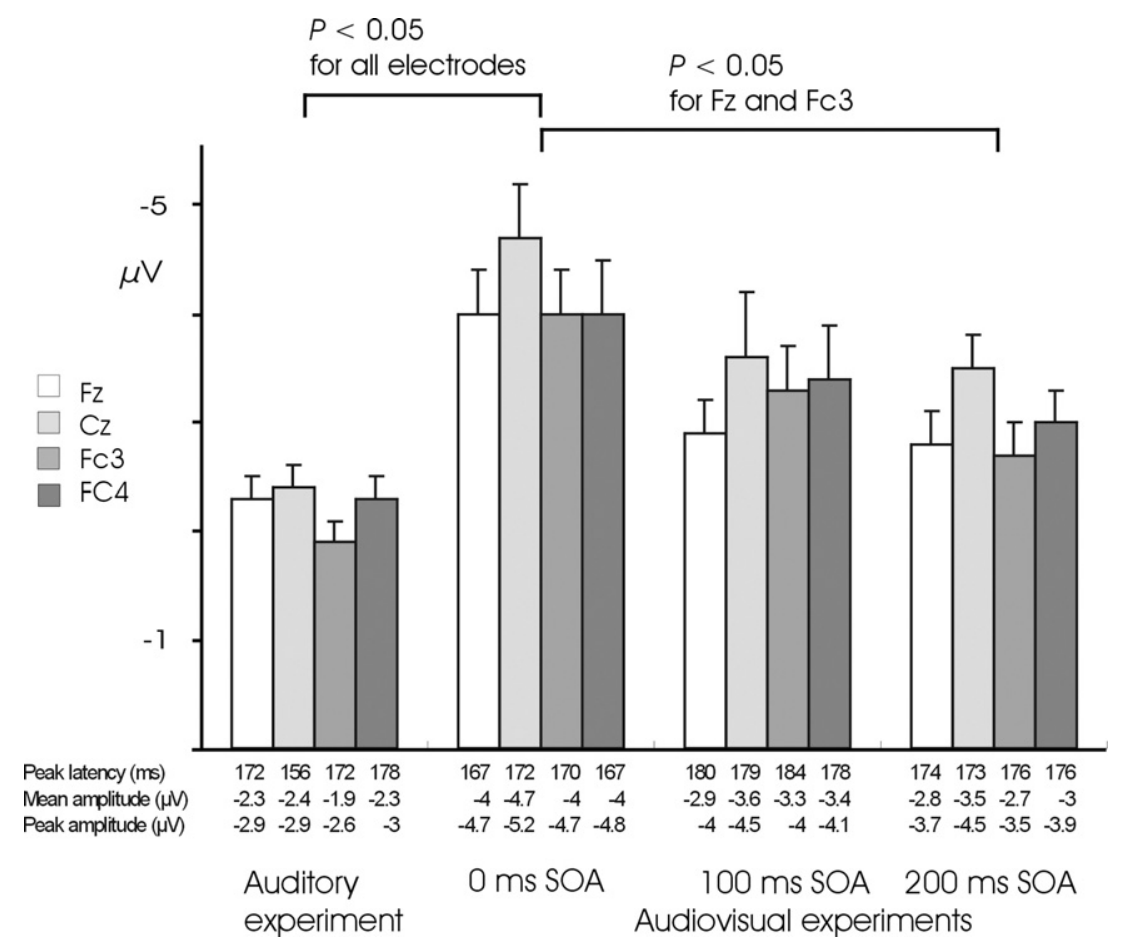

Fig. 2. Mean amplitude values over $50 \mathrm{~ms}$ around the individually assessed MMN peak with standard error for the four frontocentral electrodes Fz, $\mathrm{Cz}, \mathrm{FC} 3$ and FC4, including latency, peak and mean amplitude values.

were $169 \mathrm{~ms}$ (range: 118-206 ms) for AV_0, $177 \mathrm{~ms}$ (range: 126-214 ms) for AV_100 and $172 \mathrm{~ms}$ (range: 104-208 ms) for AV_200 after the onset of the auditory stimulus deviation (Fig. 2).

Amplitude and latency characteristics of ERP activity in the MMN time-window were first analyzed using a mixed ANOVA with two within-subject factors; stimulus type (2) and electrode sites (9). The between group variable was experiment with four levels for the four different experiments. We found an interaction between stimulus type and electrode site for mean, $F(8,416)=7.4, p<0.001$, and peak values, $F(8$, $416)=22.7, p<0.001$. We performed further analysis on four frontocentral electrodes using a 2 (stimulus type; standard versus deviant) by 4 (electrode sites) repeated-measures ANOVA with experiment as between subject variable. There was no interaction of stimulus type with electrode site for mean, $F(9$, $156)=0.2, p=0.370$, and peak values, $F(9,156)=1.2, p=0.306$. As expected, deviant stimuli elicited a significantly stronger negative ERP response than standard stimuli (main effect of stimulus type for mean amplitude, $F(1,52)=103.7 ; p<0.001$, and for peak amplitude, $F(1,52)=103.7 ; p<0.001)$. Crucially, this mismatch effect showed a significant interaction with experiment (stimulus type-by-experiment interaction for mean amplitude, $F(3,52)=6.1 ; p=0.001$, and for peak amplitude, $F(3,52)=6.1$; $p=0.01)$.

For further statistical analysis of the difference waves on the frontocentral electrodes we excluded one subject of the auditory experiment ( $Z$-scores of 2.20 for $\mathrm{Fz}, 2.15$ for $\mathrm{Cz}, 2.0$ for $\mathrm{FC} 3$ and 2.1 for FC4) and one subject of AV_100 ( $Z$-scores of -2.23 for $\mathrm{Fz},-2.6$ for $\mathrm{Cz},-2.8$ for FC3 and -2.3 for FC4). Thirteen subjects were preserved in the auditory experiment ( 10 females, range $18-33$, mean age 21.84 years). Twelve subjects were preserved in AV_100 (nine females, range 19-29, mean age 22.61 years).

A one-way ANOVA on mean values revealed a significant effect of experiment in all four electrodes, $F(3)=4.9, p=0.004$ for $\mathrm{Fz}, \mathrm{F}(3)=4.1, p=0.011$ for $\mathrm{Cz}, F(3)=5.9, p=0.001$ for $\mathrm{FC} 3$ and $F(3)=3.7, p=0.018$, for $\mathrm{FC} 4$, the same result was found for peak area values, $F(3)=4.4, p=0.008$ for $F z, F(3)=4.6$, $p=0.007$ for $\mathrm{Cz}, F(3)=5.6, p=0.002$ for $\mathrm{FC} 3$ and $F(3)=3.3$, $p=0.027$, for FC4. The MMN was stronger in the audiovisual experiments in comparison with the $\mathrm{MMN}$ in the auditory experiment (Fig. 2). Post hoc $t$-tests (Bonferroni corrected) on mean area values showed that the effect of experiment was due to a difference between the auditory experiment and AV_0, $p<0.001$ for $\mathrm{Fz}, p=0.003$ for $\mathrm{Cz}, p<0.001$ for FC3, $p=0.006$ for FC4, the same is true for peak values, $p=0.002$ for $\mathrm{Fz}, p=0.001$ for $\mathrm{Cz}, p<0.001$ for FC3, $p=0.004$ for FC4. For electrodes $\mathrm{Fz}, p=0.045$, and $\mathrm{CP} 3, p=0.030$, we also found a difference between AV_0 and AV_200. For all frontocentral electrodes the MMN in AV_0 was significantly larger than the MMN in the auditory experiment. For Fz and CP3, the MMN in AV_0 was significantly larger than the MMN in AV_200. To test whether the decrease in MMN amplitude was linear over the three audiovisual experiments we performed a polynomial contrast with the four frontocentral electrodes as a within-subject factor. This analysis revealed a significant linear contrast for both mean, $p=0.026$, and peak values, $p=0.045$.

Finally, a repeated measures ANOVA with the four frontal electrodes as a within-subject factor and experiment as a between subject factor was performed to test for an effect of latency. There was no interaction between latency and elec- 
trodes, $F(9,147)=1.2, p=0.307$, and no main effect of latency, $F(3,49)=.36, p=0.781$.

To summarize, we used the MMN to investigate the automaticity and timing characteristics of the influence of letters on the processing of speech-sounds. We observed the influence of letters on the difference wave evoked in an auditory oddball paradigm with speech-sounds and investigated the influence of stimulus onset asynchrony. In a purely auditory experiment, a typical auditory MMN was shown. We compared the auditory MMN with the negativity in the difference waves evoked in the audiovisual experiments (audiovisual MMN) and found a general enhancement of the amplitude, decreasing linearly with increasing SOA.

We hypothesized early and automatic integration of letters and speech-sounds. When simultaneously presented with the speech-sound, we found a clear influence of the letter on the MMN evoked by the deviant speech-sound; an enhancement of the MMN amplitude. This enhancement might be explained by the learned letter/speech-sound association, causing a double deviation in the audiovisual experiments: the deviant speechsound differs from the standard speech-sound and the letter on the screen. The MMN is typically evoked between 100 and $200 \mathrm{~ms}$ after stimulus onset [10,14]. This indicates that the influence of the letter on the ERP-difference wave reflecting speech-sound processing is established early in the processing of the speech-sound. Since the MMN is known to be evoked automatically [9], and there were no task requirements related to the letter or speech-sound stimuli, our findings furthermore suggest that the integration of letters and speech-sounds occurs automatically. It remains though to be investigated to what extent a neutral (non-linguistic) visual stimulus might influence the MMN evoked by a deviant speech-sound.

As mentioned before, we observed an effect of SOA within the audiovisual experiments. There was a linear trend of decreasing enhancement of the audiovisual MMN amplitude with increasing SOA. In Fz and FC3, the MMN in AV_0 differs significantly from the $\mathrm{MMN}$ in $\mathrm{AV}_{-} 200$. Since the $\mathrm{MMN}$ at AV_100 did not differ significantly from the MMN in the auditory experiment, we suggest that even $100 \mathrm{~ms} \mathrm{SOA}$ is enough to prevent complete processing of letters and speech-sounds as compounds. A similar effect of timing in the auditory association cortex was found in a previous fMRI study by our group [16]. This region is assumed to be the location of the main neural generators of the MMN [1,7], with additional frontal generators. The consistent findings from different methods (ERP and fMRI) strengthen the hypothesis that temporal proximity is a critical factor for cross-modal integration of letters and speechsounds [16]. Apparently, once arbitrarily related cross-modal associations become over-learned, integration is subject to very basic rules such as temporal proximity [8]. The narrow timewindow of integration might be a consequence of the rigidity and inflexibility of the letter/speech-sound integration process. Consistently, this integration process seems to fulfill the main criteria for automatic processing in general as formulated by Schneider and Chein [13]; letter/speech-sound integration is also described as fast, robust to distractors and requiring low effort.
The MMN is suggested to be influenced by attention in the context of an active task [11]. In the present experiment, the active task in the audiovisual experiments only served as focusing control and was completely unrelated to the processing of the letter and speech-sound stimuli. Moreover, the linear decrease of the audiovisual MMN amplitude with increasing SOA cannot be explained by the presence or absence of the focusing task, since the task was the same in all audiovisual experiments. Furthermore, at all four frontocentral electrodes the MMN at AV_200 (focusing task) was no longer significantly higher in comparison with the MMN in the auditory experiment (no focusing task). This indicates that the focusing task is not exclusively responsible for the enhancement of the MMN.

It is still possible that the presence of letters involuntary drew attention to the auditory stimuli. However, in absence of active task demands with regard to the letter and speech-sound stimuli, such an involuntary switch of attention might still be interpreted as automatic. Our suggestion is in line with our previous fMRI results of interactions between letters and speech-sounds in a completely passive design [16,17].

Considering possible contamination of the auditory evoked potentials by visual evoked potentials we checked the brain waves in the audiovisual experiments before and after the onset of the auditory stimulus (Fig. 1). Prior to auditory stimulus onset, the presented letter was identical in the standard and deviant conditions and accordingly elicited comparable visual evoked responses, resulting in the absence of a difference potential prior to auditory stimulus onset in AV_100 and AV_200. After auditory stimulus onset, the morphology of the evoked responses is comparable across the three AV experiments: including a P1N1-P2 and a sustained negativity (both in the standard and the deviant conditions). This similarity in evoked responses indicates the absence of a general modulation by overlapping visual and auditory evoked potentials at frontocentral electrodes.

In conclusion, we found a clear effect of letters on the processing of speech-sounds as reflected in the MMN. This result indicates that letter/speech-sound pairs are processed early and automatically as compound stimuli in normally reading adults. The present study provides a method to non-invasively investigate automatic letter/speech-sound integration, which might be applied in research on children of all ages. Schneider and Chein's [13] criteria imply that automaticity is an effect of extended training. In future research, it will therefore be interesting to investigate the emergence of automaticity of letter/speech-sound associations in beginning readers.

\section{Acknowledgments}

This work was supported by grant 608/002/2005 of the Dutch Board of Health Care Insurance (College voor Zorgverzekeringen) awarded to L.B. We thank Hanne Poelmans and Vera Blau for assistance in data acquisition.

\section{References}

[1] K. Ahlo, Cerebral generators of mismatch negativity (MMN) and its magnetic counterpart (MMNm) elicited by sound changes, Ear Hear. 16 (1995) $38-51$ 
[2] L. Blomert, Dyslexie in Nederland - theorie, praktijk en beleid (Dyslexia in The Netherlands - Theory, Practice and Policy), Nieuwezijds Publishers, Amsterdam, 2005.

[3] P. Boersma, D. Weenink, Praat 4.0: A System for Doing Phonetics with the Computer, Universiteit van Amsterdam, 2002.

[4] A. Dijkstra, R. Schreuder, U.H. Frauenfelder, Grapheme context effects on phonemic processing, Lang. Speech 32 (1989) 89-108.

[5] L.C. Ehri, Development of sight word reading: phases and findings, in: M.J. Snowling, C. Hulme (Eds.), The Science of Reading: A Handbook, Blackwell publishing, Oxford, 2005, pp. 135-154.

[6] D. Froyen, N. Van Atteveldt, M. Bonte, L. Blomert, (manuscript in preparation).

[7] M.H. Giard, F. Perrin, J. Pernier, P. Bouchet, Brain generators implicated in the processing of auditory stimulus deviance: a topographic event-related potential study, Psychophysiology 27 (1990) 627-640.

[8] M.A. Meredith, J.W. Nemitz, B.E. Stein, Determinants of multisensory integration in superior colliculus neurons. I. Temporal factors, J. Neurosci. 7 (1987) 3215-3229.

[9] P.P. Näätänen, H. Tiitinen, D. Jiang, K. Alho, Attention and mismatch negativity, Psychophysiology 30 (1993) 336-350.

[10] R. Näätänen, The perception of speech-sounds by the human brain as reflected by the mismatch negativity (MMN) and its magnetic equivalent (MMNm), Psychophysiology 38 (2001) 1-21.
[11] R.D. Oades, A. Dittmann-Balcar, Mismatch negativity (MMN) is altered by directing attention, Neuroreport 6 (1995) 1187-1190.

[12] T. Raij, K. Uutela, R. Hari, Audiovisual integration of letters in the human brain, Neuron 28 (2000) 617-625.

[13] W. Schneider, J.M. Chein, Controlled \& automatic processing: behavior, theory and bialogical mechanisms, Cogn. Sci. 27 (2003) 525559.

[14] E. Schröger, Measurement and interpretation of the mismatch negativity, Behav. Res. Methods Instrum. Comput. 30 (1998) 131-145.

[15] M.J. Snowling, The development of grapheme-phoneme correspondence in normal and dyslexic readers, J. Exp. Child Psychol. 29 (1980) 294 305.

[16] N. Van Atteveldt, E. Formisano, L. Blomert, R. Goebel, The effect of temporal asynchrony on the multisensory integration of letters and speech-sounds, Cereb. Cortex 13 (2007) 962-974.

[17] N. Van Atteveldt, E. Formisano, R. Goebel, L. Blomert, Integration of letters and speech-sounds in the human brain, Neuron 43 (2004) 271282.

[18] F.R. Vellutino, J.M. Fletcher, M.J. Snowling, D.M. Scanlon, Specific reading disability (dyslexia): what have we learned in the past four decades? J. Child Psychol. Psychiatry 45 (2004) 2-40. 